
\title{
25 Research Soure \\ The Need for a Broad Differential: Intramedullary Neurosarcoidosis Case Report
}

\section{Diego Dereck Luy ( $\square$ ddl15@pitt.edu )}

UPMC: University of Pittsburgh Medical Center https://orcid.org/0000-0001-6507-0884

\section{Daniel Tonetti}

UPMC: University of Pittsburgh Medical Center

\section{Peter Carlos Gerszten}

UPMC: University of Pittsburgh Medical Center

\section{Research Article}

Keywords: Enhancing Intramedullary Lesion, Intramedullary Neurosarcoidosis, Neurosarcoidosis, Cervical Syrinx, Case Report

Posted Date: October 19th, 2021

DOI: https://doi.org/10.21203/rs.3.rs-555679/v1

License: (c) (1) This work is licensed under a Creative Commons Attribution 4.0 International License. Read Full License

Version of Record: A version of this preprint was published at Neurological Sciences on February 6th, 2022. See the published version at https://doi.org/10.1007/s10072-022-05925-z. 


\section{Abstract}

\section{Background}

Sarcoidosis, an idiopathic multisystem inflammatory disorder, involves the nervous system in as few as $5-15 \%$ of cases. We aim to detail how a rare case of intramedullary neurosarcoidosis spinal-cord lesion, present in less than $1 \%$ of sarcoidosis cases, presented with features mimicking a neoplasm.

\section{Methods}

Retrospective chart review was performed to obtain pertinent details regarding history and examination, pathological findings, and treatment course.

\section{Case Presentation}

We report a case of intramedullary sarcoidosis involving the cervical and thoracic spinal cord with syringomyelia, which presented as subacute neck pain, intermittent leg paresthesias, and difficult micturition. Historically, a spinal syrinx with concern for neoplasm has led surgeons to decompress the spinal cord for certain enhancing intramedullary lesions, which is unnecessary for neurosarcoidosis. Immunosuppressant treatment resulted in symptomatic resolution without the need for spinal cord biopsy or syrinx decompression in this case.

\section{Conclusions}

Expansile contrast-enhancing intramedullary lesions, most commonly neoplastic, may instead be nonneoplastic etiologies mimicking neoplastic features; therefore, it is the responsibility of any surgeon to maintain a broad differential diagnosis in the absence of a confirmed pathology.

\section{Introduction}

Sarcoidosis, an idiopathic inflammatory disorder, involves the nervous system (neurosarcoidosis) in 5$15 \%$ of cases. ${ }^{1,2}$ Neurosarcoidosis commonly impacts the hypothalamus, pituitary gland, and cranial nerves. Intramedullary spinal neurosarcoidosis is exceptionally rare, less than $1 \%$ of cases. ${ }^{3}$ We report a patient with subacute neck pain, intermittent leg paresthesias, and difficult micturition with intramedullary contrast-enhancing lesions and syringomyelia who was diagnosed and treated successfully with immunosuppressants resulting in complete symptomatic and radiographic resolution, highlighting the responsibility of the neurosurgeon to maintain broad differential in management of enhancing spinal cord lesions.

\section{Methods}


Retrospective chart review was completed to obtain details for this report. Consent was provided for retrospective chart review to construct the manuscript and for publication of the manuscript.

\section{Case Presentation}

\section{History and Examination}

31-year-old man with no medical history presented with 3-months of neck pain, intermittent leg paresthesias, and 2 weeks of intermittent difficult micturition. Physical examination revealed positive Hoffman's reflexes and mildly diminished pinprick sensation in his lower extremities. Bladder scan revealed $>1000 \mathrm{~mL}$ of retained urine.

\section{Findings and Treatment Course}

T1-weighted gadolinium-enhanced MRI of the entire spine showed two well-defined, contrast-enhancing lesions within cervical (Fig. 1A) and thoracic (Fig. 1B) spinal cord, with associated syringomyelia. Broad differential diagnosis of spinal intramedullary enhancing lesions includes tumors (gliomas, lymphomas, hemangiomas, ependymomas), vascular lesions (cavernous malformation, dural arteriovenous fistulae), demyelinating and/or inflammatory causes (multiple sclerosis, transverse myelitis, sarcoidosis, abscesses). Laboratory assessments and serum ACE level were within normal limits.

Contrast-enhanced full-body screening CT revealed moderate bilateral mediastinal lymphadenopathy. After careful assessment of the risk profile associated with mediastinal lymph node biopsy versus spinal cord biopsy, he underwent endobronchial ultrasound-guided fine-needle aspiration of an enlarged mediastinal lymph node. Histopathological examination revealed non-caseating granulomas with multinucleated giant cells (Fig. 1C,), suggesting intramedullary neurosarcoidosis. Treatment with systemic glucocorticoids, infliximab, and methotrexate resulted in complete resultant neurologic improvement to baseline function within two weeks. Follow-up MRI four months after initiating treatment showed no residual enhancement and complete resolution of the syrinx (Fig. 2).

\section{Discussion}

Sarcoidosis, an idiopathic multisystem granulomatous disorder, usually affects lungs, lymph nodes, eyes, and skin; however, it may also affect the nervous system in $5-15 \%$ of cases. ${ }^{4,5}$ The prototypical patient is a 30-40-year-old African American female. ${ }^{3}$ Approximately half of patients present without prior sarcoidosis diagnosis. ${ }^{6}$ Neurosarcoidosis is known to mimic neoplasms including ependymoma, astrocytoma, or gliomas; demyelinating diseases including multiple sclerosis or acute disseminated encephalomyelitis; transverse myelitis; and fungal infections. ${ }^{3,7}$ Historically, concern for neoplasm has 
persuaded surgeons to decompress the spinal cord in certain enhancing intramedullary lesions, particularly with accompanying syrinx, resulting in misdiagnosis and inadequate management. ${ }^{3,8}$

We describe a rare case of neurosarcoidosis involving contrast-enhancing cervical and thoracic spinal cord lesions with syringomyelia. Findings of cervical and thoracic enhancing masses (Fig. 1A, B) suggested primary malignancy including ependymoma, astrocytoma, or spinal hemangioblastoma. Sarcoidosis, lymphoma, or nonspecific infectious/inflammatory processes were considered more likely after noting mediastinal and hilar lymphadenopathy, despite negative serum ACE levels. Mediastinal lymph node fine-needle aspiration revealed non-caseating granulomas with multinucleated giant cells (Fig. 1C), consistent with sarcoidosis, and prompt treatment with systemic glucocorticoids, infliximab, and methotrexate resulted in rapid neurologic improvement and resolution of enhancing spinal cord masses and syringomyelia (Fig. 2). A thoughtful approach for enhancing intramedullary spinal cord lesions is the neurosurgeon's responsibility and may obviate the need for decompressive surgery despite the presence of syrinx and/or neurologic deficit. This case report is inherently limited due to retrospective review of clinically relevant patient presentation, treatment, and follow-up. The limitations were minimized through an in-depth review of the patient's medical record.

\section{Conclusion}

A rare case of neurosarcoidosis involving contrast-enhancing cervical and thoracic spinal cord lesions with syringomyelia supports that expansile contrast-enhancing intramedullary lesions, most commonly neoplastic, may instead be non-neoplastic etiologies mimicking neoplastic features. Therefore, it is the responsibility of any surgeon to maintain a broad differential diagnosis in the absence of a confirmed pathology.

\section{Declarations}

\section{i. Funding}

This research received no specific grant from any funding agency in the public, commercial or not-forprofit sectors.

\section{ii. Conflicts of interest/Competing interests}

The authors have no personal or institutional interest with regards to the authorship and/or publication of this manuscript.

\section{iii. Ethics approval}


University of Pittsburgh Institutional Review Board authorization: PRO08120394. Consent was provided for chart review to construct the manuscript and for publication of the manuscript.

\section{iv. Consent to participate}

Consent was provided for retrospective chart review to construct the manuscript and for publication of the manuscript.

\section{v. Consent for publication}

Consent was provided for retrospective chart review to construct the manuscript and for publication of the manuscript.

\section{vi. Availability of data and material (data transparency)}

Not applicable

\section{vii. Code availability (software application or custom code)}

Not applicable

\section{viii. Authors' contributions}

DDL and DT were involved in the design and conception of this manuscript. DDL performed the literature search and acquired the data. DDL and DT compiled the primary manuscript. DDL and DT compiled the figures. DT and PCG critically revised the manuscript. All authors have approved the manuscript as it is written.

\section{References}

1. Hoitsma E, Faber CG, Drent M, Sharma OP. Neurosarcoidosis: a clinical dilemma. Lancet Neurol. 2004;3(7):397-407. 10.1016/s1474-4422(04)00805-1.

2. Stern BJ, Krumholz A, Johns C, Scott P, Nissim J. Sarcoidosis and Its Neurological Manifestations. Arch Neurol. 1985;42(9):909-17. 10.1001/archneur.1985.04060080095022.

3. Saadi A, Rajashekara S. Intramedullary spinal neurosarcoidosis. Radiol Case Rep. 2012;7(4):739. 10.2484/rcr.v7i4.739.

4. Kidd DP. Sarcoidosis of the central nervous system: clinical features, imaging, and CSF results. J Neurol. 2018;265(8):1906-15. 10.1007/s00415-018-8928-2. 
5. Padooru KR, Sen M. Intramedullary spinal cord involvement: a rare presentation of sarcoidosis. Int Med Case Rep J. 2019;12:199-203. 10.2147/imcrj.S212229.

6. Kumar N, Frohman EM. Spinal neurosarcoidosis mimicking an idiopathic inflammatory demyelinating syndrome. Arch Neurol. 2004;61(4):586-9. 10.1001/archneur.61.4.586.

7. Chang BL, Kuo HC, Chu CC, Huang CC. Spinal neurosarcoidosis. Acta Neurol Taiwan. 2011;20(2):142-8.

8. Kwon DH, Lee SH, Kim ES, Eoh W. Intramedullary sarcoidosis presenting with delayed spinal cord swelling after cervical laminoplasty for compressive cervical myelopathy. J Korean Neurosurg Soc. 2014;56(5):436-40. 10.3340/jkns.2014.56.5.436.

\section{Figures}
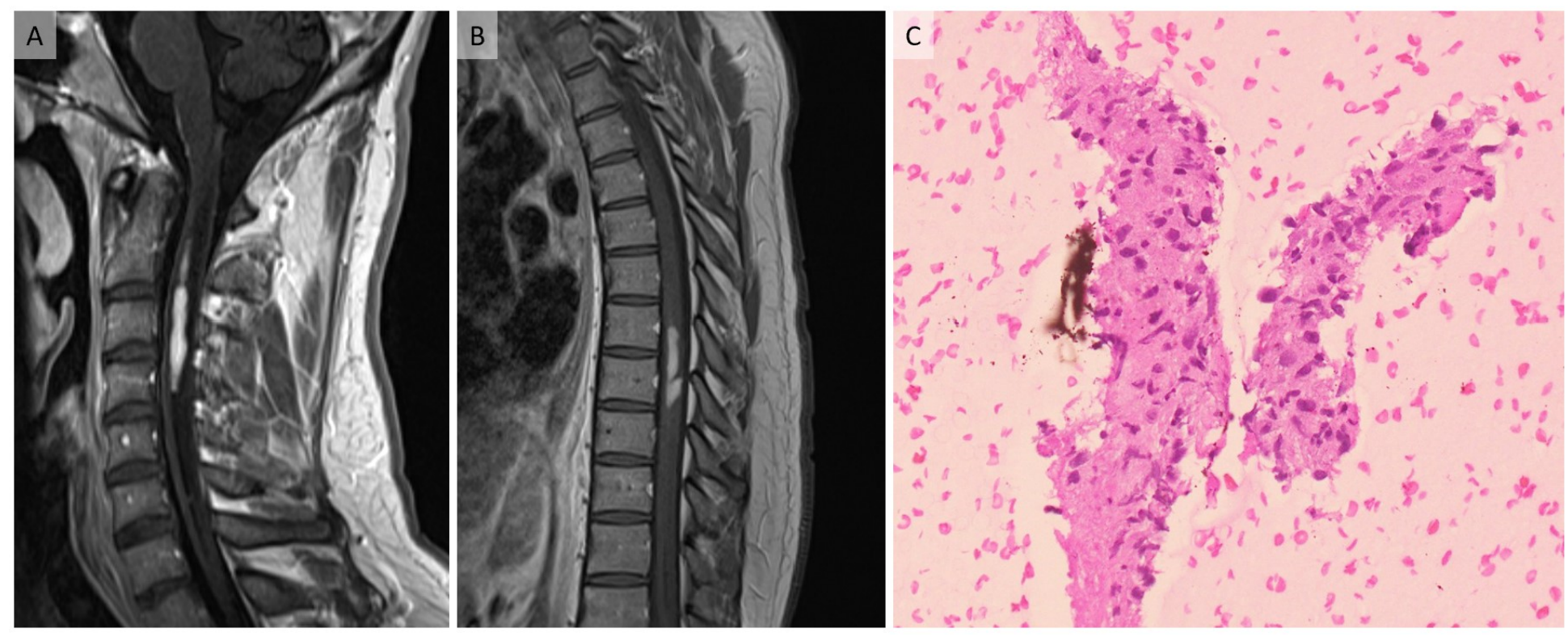

\section{Figure 1}

Pre-treatment T1-weighted gadolinium-enhanced Sagittal MRI showing contrast-enhancing lesions within the cervical (panel A) and thoracic (panel B) spinal cord, associated syringomyelia, and slide of hematoxylin and eosin-stained fine-needle aspiration biopsy of mediastinal lymph nodes showing noncaseating granulomas with multinucleated giant cells (panel C). 


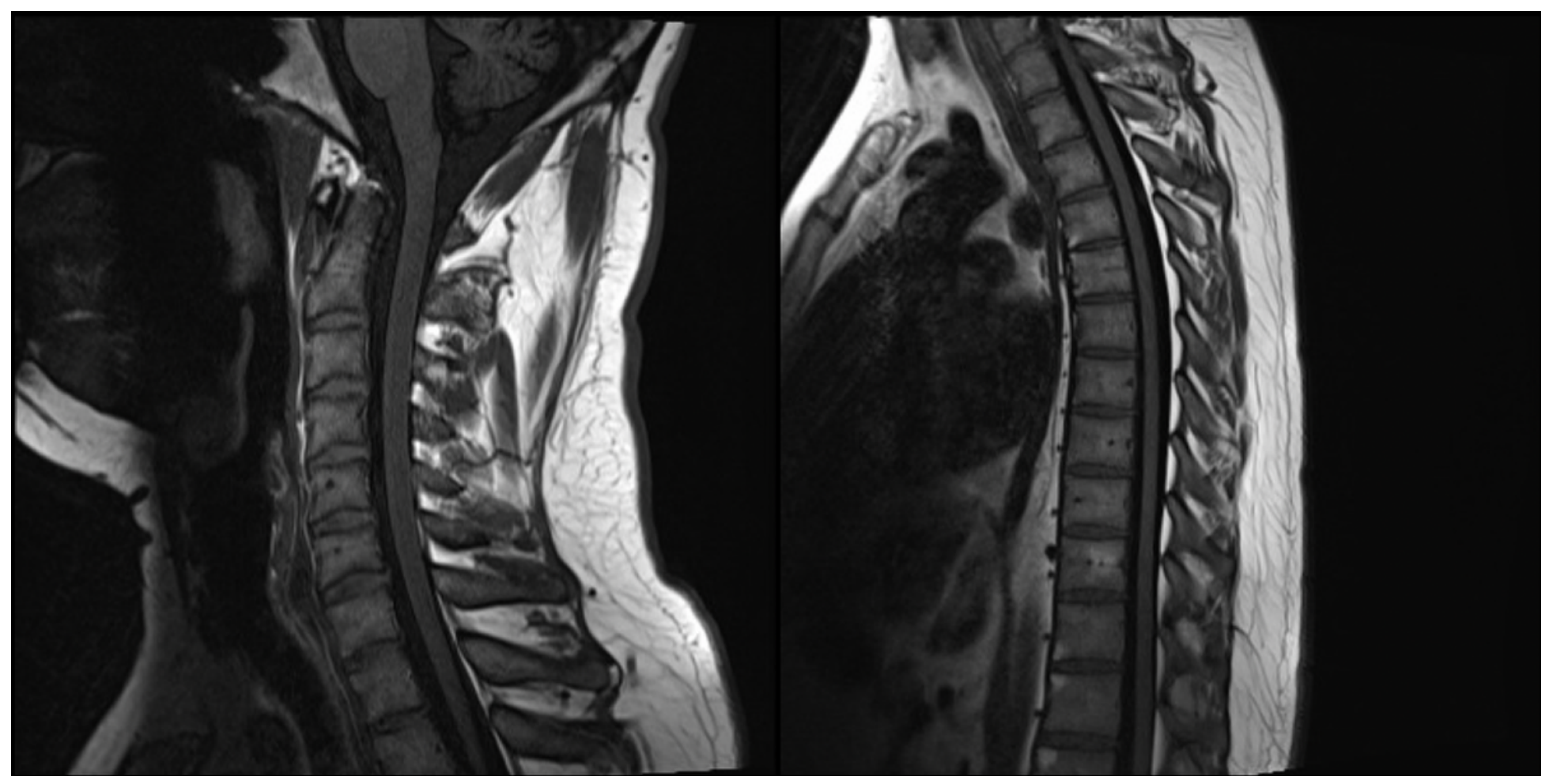

\section{Figure 2}

Post-treatment T1-weighted gadolinium-enhanced Sagittal MRI showing normalization of cervical syrinx (left) and no residual enhancement (right).

\section{Supplementary Files}

This is a list of supplementary files associated with this preprint. Click to download.

- CAREchecklist.pdf 